\title{
Ferias Científicas como Escenarios de Motivación e Interés por la Ciencia en Estudiantes Chilenos de Educación Media de la Región Metropolitana
}

\author{
Luz V. Oppliger ${ }^{(1) \star}$, Paula Nuñez ${ }^{(1)}$ y Stefan Gelcich ${ }^{(1,2)}$ \\ (1) Centro de Ecología Aplicada y Sustentabilidad, (CAPES), Departamento de Ecología, Facultad de Ciencias \\ Biológicas, Pontificia Universidad Católica de Chile, Casilla 114-D, Santiago, Chile. (e-mail: valeriaoppliger@gmail.com; \\ pnnunez@uc.cl) \\ (2) Núcleo Milenio Centro para el Impacto Socioeconómico de las Políticas Ambientales (CESIEP), Pontificia \\ Universidad Catolica de Chile, Santiago, Santiago, Chile (e-mail: sgelcich@bio.puc.cl)
}

* Autor a quien debe ser dirigida la correspondencia.

Recibido Feb. 11, 2019; Aceptado Abr. 5, 2019; Versión final Jun. 17, 2019, Publicado Dic. 2019

\begin{abstract}
Resumen
Este estudio evaluó la percepción a corto plazo de una feria científica sobre la motivación, la complejidad y el interés por la ciencia en estudiantes chilenos de educación media. La feria consistió en puestos de diferentes formatos presentados por estudiantes de posgrado. Los asistentes del evento fueron estudiantes de educación media de entre 14-18 años, pertenecientes a todos los rangos socioeconómicos de la Región Metropolitana de Chile. El estudio consistió en la realización de una encuesta previa a la entrada de la feria, y otra posterior, a la salida. Los resultados muestran que la motivación y el interés por la ciencia aumentaron después de la experiencia, mientras que la percepción de complejidad de dicha materia disminuyó. A partir de los resultados de este estudio se concluye que las ferias científicas tienen un impacto o impresión positiva en los asistentes, independiente de su género o nivel socioeconómico. Estos resultados concuerdan parcialmente con estudios similares realizados en otros países.
\end{abstract}

Palabras clave: feria científica; percepción de la ciencia; complejidad de la ciencia; motivación por la ciencia; carrera científica

\section{Scientific Fairs as Scenarios for Motivating Interest in Science in Chilean High School Students from the Metropolitan Region}

\begin{abstract}
This study evaluated the short-term perception of a scientific fair on motivation, complexity and interest in science in Chilean high school students. The fair consisted of stands of different formats presented by graduate students. The attendees of the event were high school students that ranged from 14-18 years from all socioeconomic ranks of the metropolitan region of Chile. A survey was carried out prior to the entrance to the fair, and a later one, upon departure. The results show that motivation and interest in science increased after the experience, while the perception of the complexity of science decreased. Results of this study suggest that science fairs have a positive impact or impression on attendees, and that it does not depend on gender or socioeconomic level. These results are partially consistent with similar studies carried out in other countries.
\end{abstract}

Keywords: scientific fair; perception of science; science complexity; motivation for science; scientific career 


\section{INTRODUCCIÓN}

La divulgación de la ciencia es una disciplina que genera espacios de encuentro entre la comunidad científica y el público no especializado. El ejercicio de la divulgación de la ciencia es importante para el fortalecimiento del desarrollo científico, la generación de confianza entre la sociedad civil y la comunidad de especialistas, y el enriquecimiento de la cultura y el pensamiento crítico (Fariña et al., 2018). Se ha reportado que la divulgación científica desarrolla sentido de pertenencia y motivación por la misma (Luehmann y Markowitz, 2007), aumenta el interés por estudiar carreras científicas (Markowitz, 2004), incrementa el desarrollo de las ciencias y su financiamiento (Gibson y Chase, 2002), además de proveer nuevas oportunidades a la ciudadanía al crear una sociedad mejor informada y con mayor cultura científica (Ray, 1999; Luehmann, 2009). No menor es el hecho de que la ciencia se financia, principalmente, con fondos públicos, como es el caso de Chile, y el conocimiento generado debiera ser accesible a todo miembro de la sociedad.

Tradicionalmente, se ha asumido que la enseñanza de las ciencias debe ser incorporada, en principio, en la sala de clases, a través del programa escolar que determina cada país. Sin embargo, hallazgos recientes cuestionan esta antigua creencia, sugiriendo que gran parte de los conocimientos en ciencias se adquieren fuera del sistema escolar tradicional (Falk y Dierking, 2010). Por ejemplo, en Estados Unidos el estudio de Falk y Dierking muestra que sólo alrededor del cinco por ciento de la vida de un estadounidense se desarrolla en el aula, mientras que sólo una pequeña fracción de eso se dedica a la enseñanza de la ciencia. Este análisis sugiere que la mejor manera de aumentar la comprensión pública de la ciencia es llegar a las personas durante el otro 95 por ciento de su vida (Falk y Dierking, 2010). Dentro de la divulgación de la ciencia, existen numerosas y diversas iniciativas tales como charlas, congresos escolares, laboratorios abiertos, exposiciones, talleres y ferias científicas. Todas las anteriores podrían clasificarse como iniciativas de educación o aprendizaje informal, ya que gran parte, o la mayoría, ocurren fuera de la sala de clases formal (Jeffs y Ord, 2018).

Frente a la oportunidad que representan los sistemas de educación no formal en la alfabetización científica, es pertinente evaluar estas actividades, así como también sus metodologías. Esta evaluación es fundamental para evidenciar qué tan efectivas son estas actividades en transmitir los conocimientos, conceptos y habilidades que se quieren transmitir, además de identificar lecciones sobre enfoques prometedores para futuras inciativas (Diamond et al., 2016). Algunos de los elementos de las iniciativas que han sido evaluados son: conocimientos, pertenencia, habilidades, comportamiento, actitudes, intenciones, entre otros. Estas evaluaciones pueden ser de tipo cualitativas o cuantitativas, luego de la exposición de los participantes a las actividades (Stern et al., 2014).

Una modalidad de divulgación científica de larga tradición a nivel mundial son las ferias científicas. Las Ferias de Ciencia y Tecnología son actividades programadas, en las que los estudiantes son los protagonistas, ya que plantean problemas, desarrollan proyectos de investigación en diversas temáticas científicas y aplicaciones, además de presentar y comunicar sus hallazgos a las comunidades (Retana et al., 2018). También participan profesores e instituciones educativas, pero en un lugar secundario. La génesis de estas ferias varía mucho de un lugar a otro, pues dependen de la institución que convoca y de los objetivos propuestos (Chaparro et al., 2018).

Dentro de la gran diversidad de Ferias de Ciencia y Tecnología que se realizan, existe dificultad para implementar una clasificación estándar. Entre los criterios que destacan para poder clasificarlas encontramos: formato de los trabajos, temática, ubicación, organizadores, ámbito de radio de acción, público convocado, línea curricular o progama educativo, utilización de método científico, aforo, financiación, objetivos, enfoque, apoyo al profesorado, participación de profesionales, incentivos o premios para los participantes, y presencia de actividades complementarias (Chaparro et al., 2018).

Cuando se diseña una feria se debe definir el público al que irá dirigida, para luego fijar los objetivos y organizar una adecuada logística del encuentro. Los públicos pueden ser el alumnado, el profesorado, personal investigador, empleados públicos, empresas/profesionales, familias, o colectivos en riesgo de exclusión social. Además de definir el público se deben fijar objetivos pedagógicos, tanto para participantes como para asistentes. En este aspecto es importante diferenciar entre el alumnado que participa en el evento de forma activa, exponiendo y presentando su proyecto, y aquellos que asisten como público. En el caso de los participantes, los objetivos más frecuentes son: aumentar el interés y competencias en ciencias y otras áreas del conocimiento, desarrollar pensamiento crítico a través del método científico, mejorar el aprendizaje, comunicarse con la sociedad, fomentar la cultura científica y crear vocación. Por otro lado, en el caso de los asistentes los objetivos más comunes son: aumentar el interés por la ciencia y tecnología, incrementar conocimientos a través de actividades lúdicas, sensibilizar de la importancia y cotidianidad de la ciencia, y fomentar la creatividad, el espíritu innovador y emprendedor (Chaparro et al., 2018). 
En el desarrollo y ejecución de las ferias se reflejan factores cognitivos, afectivos y sociales de la educación científica escolar, y también muestran el nivel de desarrollo profesional del profesorado asociado y la cultura científica de las familias y visitantes (Retana et al., 2018). Entre sus beneficios como herramienta educativa se ha descrito que las ferias científicas facilitan el desarrollo de habilidades, mejoran la concepción de fenómenos científicos, facilitan la autonomía y mejoran la autoestima de los alumnos (Bencze y Bowen, 2009; Araújo, 2015). Sin embargo, al igual que como sugirió Finnerty en 2013, se necesita investigar más sobre la calidad de las experiencias de los estudiantes en las Ferias de Ciencia y Tecnología, ya sea como participantes o asistentes de estas. Para esto es muy importante pensar qué evaluar, cómo, con qué o cuáles herramientas.

El libro verde Ferias de la Ciencia (2018) de la Fundación Española para la Ciencia y Tecnología del Gobierno de España, sugiere una completa estrategia de evaluación para ferias de Ciencia y Tecnología desde el diseño inicial de un proyecto. Como punto de partida al momento de decidir qué evaluar, esta publicación sugiere seguir un criterio de objetivos que sean específicos (Specific), medibles (Measurable), alcanzables (Achievable), relevantes (Relevant), y de duración determinada (Time-bound). Este criterio se denomina SMART (Doran, 1981). A través de él se podrían evitar errores comunes, como el establecimiento de objetivos sólo por ser medibles, pero poco relevantes, o la fijación de objetivos que son relevantes, pero no medibles.

Con objetivos claramente identificados para su medición, es necesario también analizar el nivel de impacto potencialmente alcanzable y medible según los objetivos propuestos. Los niveles de impacto que pueden alcanzarse en este tipo de eventos, de menor a mayor grado de profundidad, son: 1) de reacción o percepción, y 2) de conciencia, conocimiento y aprendizaje. Niveles mayores de impacto como cambios de comportamientos o actitudes son de largo plazo y sólo pueden ser medibles con estudios que hagan seguimientos de los sujetos de interés (Chaparro et al., 2018).

Luego, en la elección de cómo evaluar, se debe escoger entre los enfoques cuantitativos, los cualitativos y los mixtos. A grandes rasgos, las diferencias entre aproximaciones cuantitativas y cualitativas es que las primeras intentan responder a preguntas con respuestas numéricas, mientras que las segundas a preguntas más explicativas o descriptivas. Los enfoques mixtos, por su parte, tienen mezclas de ambos tipos de preguntas.

Finalmente, en la selección de herramientas de evaluación, se deben tener en cuenta las cinco opciones disponibles y bien documentadas a desarrollar para cada caso. Estas son: a) cuestionarios o encuestas, b) entrevistas, c) experiencias focales, d) observaciones y e) métricas digitales. Los cuestionarios son la herramienta de evaluación más utilizada, pues permiten desarrollar enfoques tanto cuali como cuantitativos, además de repartirlos tanto impresos como digitales.

Recientemente, Chile ha aumentado el interés por realizar divulgación científica. Esto ha sido promovido desde el año 2010 por la Comisión Nacional de Ciencia y Tecnología (CONICYT), al incorporar la divulgación como requisito de postulación a los fondos públicos otorgados en sus distintos programas. Entre las más de cinco mil actividades de divulgación que se estima se realizan anualmente a lo largo del territorio chileno, cerca de quinientos eventos corresponden a ferias científicas. Desafortunadamente, de estos eventos sólo se reporta el número de asistentes y se desconoce totalmente la calidad e impacto de las experiencias, tanto de participantes como de asistentes.

Con todo lo expuesto anteriormente, el objetivo de este estudio fue evaluar con estrategia mixta la percepción sobre la ciencia y tecnología en los asistentes de una feria científica chilena, realizada en la Pontificia Universidad Católica de Chile. Específicamente, se evaluaron: a) la motivación, b) la percepción de la complejidad de la ciencia, c) el interés de estudiar una carrera científica, d) los conceptos asociados a "ciencia" y "científico", y e) el formato de la feria misma.

\section{METODOLOGÍA}

La metodología se presenta en dos subsecciones: en una se describe la feria y sus asistentes; en la otra se detalla cómo se realizaron las evaluaciones.

\section{Feria y asistentes}

La "Feria de Divulgación Científica UC" se realizó durante tres días en el mes de mayo de 2014 en el Centro de Extensión UC, dependencia de la Pontificia Universidad Católica de Chile en Santiago para actividades culturales abiertas al público general. El evento consistió en una feria con veintinueve stands liderados por alumnos de programas de doctorado pertenecientes a cinco facultades de ciencias: Ingeniería, Medicina, Física y Astronomía, Química y Ciencias Biológicas. Los stands consistieron en: 1) modelos 3D, de distintos materiales; 2) afiches de tipo papelógrafos; 3) juegos digitales o de mesa; 4) discusiones científicas entre 
monitores del stand con asistentes y 5) experiencias de indagación, consistentes en pequeños experimentos en que los asistentes se involucran en entender un fenómeno y, por ende, en la adquisición del conocimiento (Tabla 1). Cada stand podía albergar hasta cinco asistentes por sesión y contaba con dos monitores constantes, quienes guiaron las sesiones de diez minutos.

Tabla 1: Títulos y tipos de stands de la Feria de Divulgación Científica UC: (1) Modelos 3D; (2) afiches; (3) juegos; (4) discusiones científicas; (5) experiencias de indagación.

\begin{tabular}{|c|c|}
\hline Título del stand & Tipo de stand \\
\hline Polímeros: ladrillos químicos & 1,5 \\
\hline La química de nuestro cuerpo & 3,4 \\
\hline Jugando con los estados de la materia & 5 \\
\hline Luz, química y jacción! & 5 \\
\hline Los secretos del vino & 4 \\
\hline Manejo de la calidad del agua: Un desafío actual & 4 \\
\hline Construcciones seguras antiterremotos & 4 \\
\hline ¡Aprender es un juego! 1 & 3 \\
\hline ¡Aprender es un juego! 2 & 3 \\
\hline Electricidad: Desde lo más grande a lo más pequeño & 5 \\
\hline Física como ciencia básica y aplicada & 5 \\
\hline Óptica & 5 \\
\hline Clasificación de las galaxias & 3 \\
\hline Microorganismos en tu cuerpo: Una pequeña Guerra & 4 \\
\hline El hígado en acción: "La estrella de rock del cuerpo humano" & 3 \\
\hline Diente blanco, ¡no me dejes! & 3 \\
\hline Pulmones: ¿simples globos en nuestro organismo? & 5 \\
\hline Nuestro organismo en guerra: Enfermedad respiratoria, respuesta inmune y vacunas & 5 \\
\hline Introducción al mundo de los microorganismos & 1 \\
\hline La nueva era de la Biología: Biología Sintética & 3 \\
\hline Localización y función de distintas regiones del cerebro & 4 \\
\hline El sol tiene dos caras & 4 \\
\hline Drogas de abuso y adicción & 4 \\
\hline Soy de la forma que debo ser: Forma y tamaño celular & 1 \\
\hline Cambio Global en Chile & 1,4 \\
\hline Interacciones ecológicas & 3 \\
\hline "La ecología es el teatro donde actúa la Evolución " Hutchinson, 1965 & 1,4 \\
\hline Fisiología deportiva & 5 \\
\hline Síndrome metabólico & 4 \\
\hline
\end{tabular}

Los asistentes de la feria consistieron en estudiantes de educación media de distintos establecimientos educacionales de la región Metropolitana de Santiago, representando a todo el espectro de educación socioeconómico chileno: sistema público, sistema subvencionado y sistema privado. Grupos de 250 estudiantes fueron invitados a visitar la feria en sesiones de tres horas. Se estimó que un estudiante podía visitar al menos quince stands en ese período de tiempo. Cada visitante debió concurrir a la feria con una encuesta de entrada completa, la que incluía su asentimiento para usar la información en este estudio. Además, cada asistente trajo un consentimiento de su apoderado responsable firmado. En la entrada del evento, los estudiantes recibieron un mapa de la feria y un pasaporte científico que consistió en un pedazo de cartón impreso con espacios para timbres. Luego de la visita de un stand, el asistente recibió un timbre en su pasaporte. Con quince timbres, el asistente tuvo acceso a la encuesta final de la feria, la que además entraba en un sorteo de tres tablets de última generación. Con veinte timbres en el pasaporte, el asistente podía canjear un regalo que consistía en la polera de la feria.

\section{Evaluaciones}

La motivación, la percepción de complejidad sobre la ciencia, el interés de estudiar una carrera científica y la estructura de la feria se midieron con encuestas antes (de aquí en adelante denominadas a priori) y después del evento (de aquí en adelante denominadas a posteriori). Se utilizaron escalas de uno a siete, en 
las que, para motivación por la ciencia, uno correspondió a nada de entusiasmo y siete a mucho entusiasmo. Para complejidad de la ciencia, uno correspondió a muy difícil y siete a muy fácil. Para interés en seguir una carrera científica, uno correspondió a sin interés y siete a mucho interés. Las encuestas a priori, junto con el mapa y el pasaporte, se entregaron al ingresar a la feria. Las encuestas a posteriori se entregaron en un stand con un mesón y sillas, a los asistentes con al menos quince timbres en su pasaporte. Las encuestas fueron completadas autónoma y libremente por los asistentes con monitores a disposición en caso de dudas para responder.

Los elementos o formatos de los stands de la feria, correspondientes a: (1) modelos 3D, (2) afiches, (3) juegos, (4) discusiones científicas, y (5) experiencias de indagación, se evaluaron con encuestas a posteriori en escalas del uno al siete, siendo uno muy malo y siete excelente. Además, en la encuesta a posteriori se preguntó a los asistentes si recomendaban la feria a sus pares, con respuestas de SI y NO.

Finalmente, la percepción de los conceptos "ciencia" y "científico" se evaluó en pruebas a priori y a posteriori, en las que se pidió a los encuestados que escribieran tres palabras que vinieran a su mente respecto al concepto de ciencia y tres palabras para el concepto científico. Las encuestas se desarrollaron bajo la supervisión del comité de ética de estudios sociales de la Pontificia Universidad Católica de Chile. La complejidad, la motivación y el interés en estudiar una carrera científica se analizaron antes y después de la feria, en relación con el género y el tipo de institución educativa. Específicamente, se realizaron pruebas de $t$ de los asistentes para la visión general y ANOVAS de 2 vías para probar los efectos entre el género y el tiempo, y el tipo de escuela y el tiempo, para los tres parámetros probados. Se consideraron resultados significativos los valores de $\mathrm{P}<0.05$. El software Graphpad Prism (versión 6.0 para Mac OS X) se utilizó para este propósito.

Los elementos de la feria se analizaron con ANOVA de 1 vía y una prueba de comparaciones múltiples de Tukey a posteriori con el software Graphpad Prism (versión 6.0 para Mac OS X). Los resultados de percepción del estudiante para la ciencia y los científicos fueron analizados con el software Wordle. Wordle genera "palabras en nube" a partir de un texto que da importancia a las palabras que aparecen con mayor frecuencia en el texto de origen.

\section{RESULTADOS Y DISCUSIÓN}

La Feria de Divulgación Científica UC tuvo cerca de 1000 asistentes de educación media. De esos, 716 entregaron la encuesta de entrada o test a priori, y 642 respondieron la encuesta de salida 0 test $a$ posteriori. Los asistentes tenían entre 14 a 18 años, de ellos el $37 \%$ era de sexo masculino y el $63 \%$ de sexo femenino. Respecto al origen educacional, el $25 \%$ provenía de instituciones de educación pública, el $45 \%$ de educación subvencionada y el $30 \%$ de educación privada.

\section{Motivación}

El entusiasmo general por la ciencia aumentó después de la feria $(t=7.94 ; P=\langle 0.0001 ; g l=1329)$, de 5.91 +/- 1.08 antes del evento a $6.36+/-0.96$ después del evento. Esta tendencia se observó tanto en hombres como en mujeres $(F=51.86 ; P=<0.0001 ; g l=1)$ después de la feria. Los hombres subieron de $5.89+/-1.05$ a $6.17+/-1.03$, y las mujeres de $5.9+/-1.17$ a $6.47+/-0.9$. Esta tendencia también se observó para los tres tipos de instituciones educativas que alcanzaron la feria $(F=83.91 ; P=<0.0001 ; g l=1)$. Las escuelas públicas aumentaron de $5.77+/-1.13$ a $6.29+/-1.20$, mientras que las escuelas subsidiadas de $6.02+/-098$ a $6.38+/-0.94$, y las escuelas privadas de $5.8+/-1.16$ a $6.37+/-1.20$ (Tabla 2, Figura 1 ).

Tabla 2. Resultados estadísticos de las ANOVA de 2 vías para tipo de establecimiento educacional y género antes y después de la visita.

\begin{tabular}{lccccccccc}
\hline Evaluación & \multicolumn{4}{c}{ Motivación } & \multicolumn{3}{c}{ Complejidad } & \multicolumn{3}{c}{ Interés } \\
Parámetro a comparar & Gl & $\mathrm{F}$ & $\mathrm{P}$ & $\mathrm{I}$ & $\mathrm{F}$ & $\mathrm{P}$ & $\mathrm{Gl}$ & $\mathrm{F}$ & $\mathrm{P}$ \\
\hline Género & 1 & 6,776 & 0,0093 & 1 & 1,825 & 0,1769 & 1 & 16,74 & $<0,0001$ \\
Feria científica & 1 & 51,86 & $<0,0001$ & 1 & 21,53 & $<0,0001$ & 1 & 23,35 & $<0,0001$ \\
Interacción & 1 & 5,817 & 0,016 & 1 & 0,5765 & 0,4478 & 1 & 1,592 & 0,2073 \\
Establecimiento educacional & 2 & 3,515 & 0,0299 & 2 & 2,183 & 0,1131 & 2 & 2,735 & 0,0653 \\
Feria científica & 1 & 83,91 & $<0,0001$ & 1 & 26,48 & $<0,0001$ & 1 & 21,83 & $<0,0001$ \\
Interacción & 2 & 1,406 & 0,2453 & 2 & 1,6 & 0,2023 & 2 & 1,006 & 0,3659 \\
\hline
\end{tabular}




\section{Complejidad de la ciencia}

La percepción general de la complejidad de la ciencia disminuyó después de la feria $(\mathrm{t}=4.73 ; \mathrm{P}=\langle 0.0001 ; \mathrm{gl}$ $=1323$ ), de $4.32+/-1.45$ antes del evento a $4.7+/-1.49$ después del evento. Esta tendencia se observó tanto en hombres como en mujeres $(F=21.53 ; \mathrm{P}=<0.0001 ; \mathrm{gl}=1)$ después de la feria. Los hombres subieron de $4.27+/-1.52$ a $4.59+/-1.61$, y las mujeres de $4.32+/-1.43$ a $4.76+/-1.42$. Esta tendencia también se observó para los tres tipos de instituciones educativas que alcanzaron la feria $(F=26.48 ; \mathrm{P}=<0.0001 ; \mathrm{gl}=1)$. Las escuelas públicas aumentaron de $4.26+/-1.47$ a $4.85+/-1.47$, mientras que las escuelas subsidiadas de 4.28 +/- 1.44 a $4.51+/-1.60$, y las escuelas privadas de $4.36+/-1.48$ a $4.84+/-1.32$ (Tabla 2, Fig.2).

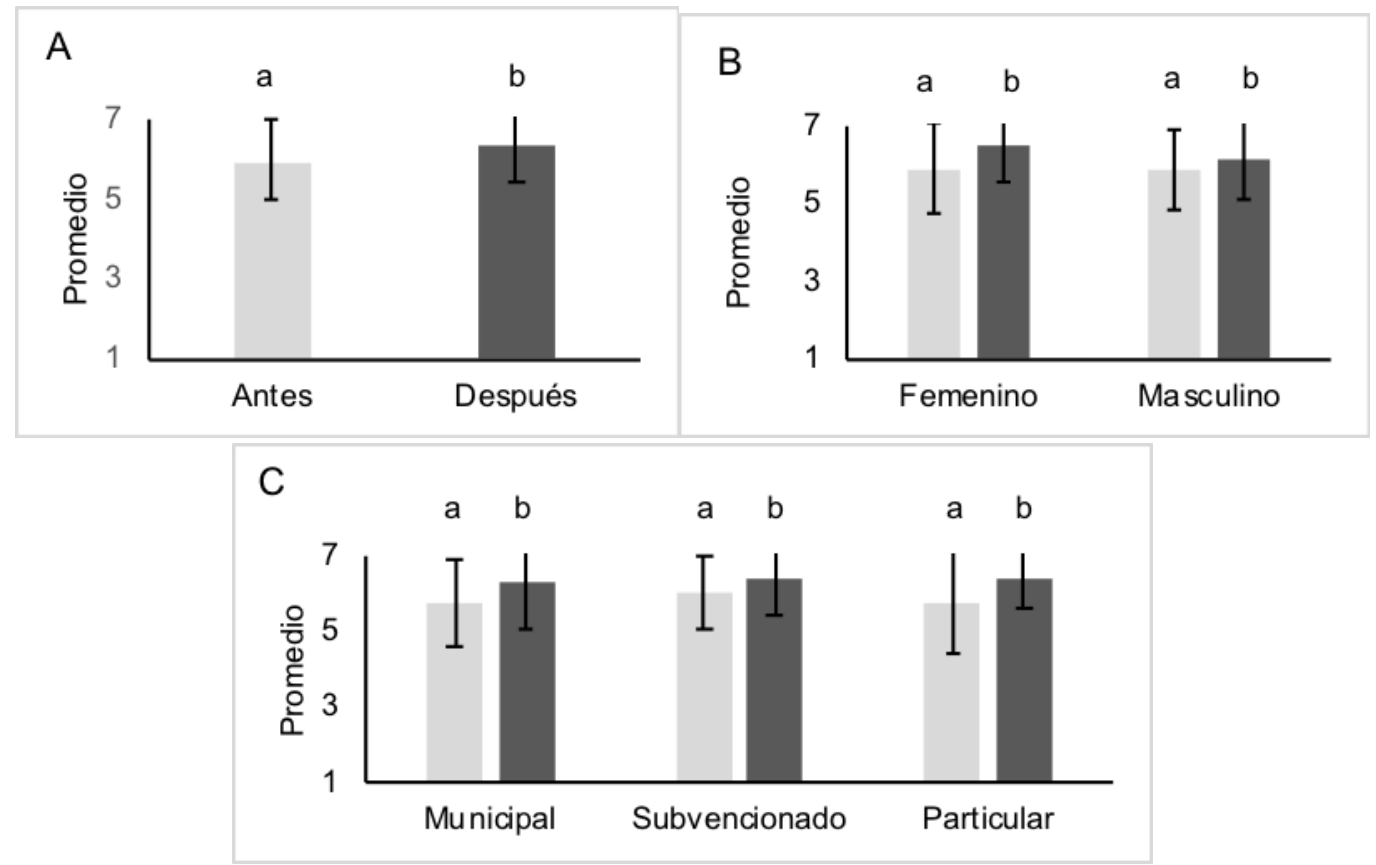

Fig. 1: Motivación declarada por los asistentes a la feria antes (gris claro) y después (gris oscuro) del evento. Resultados (A) generales, (B) por género, y (C) tipo de establecimiento educacional. La escala representa la motivación por la ciencia, donde 1 = nada de entusiasmo y 7 = mucho entusiasmo.

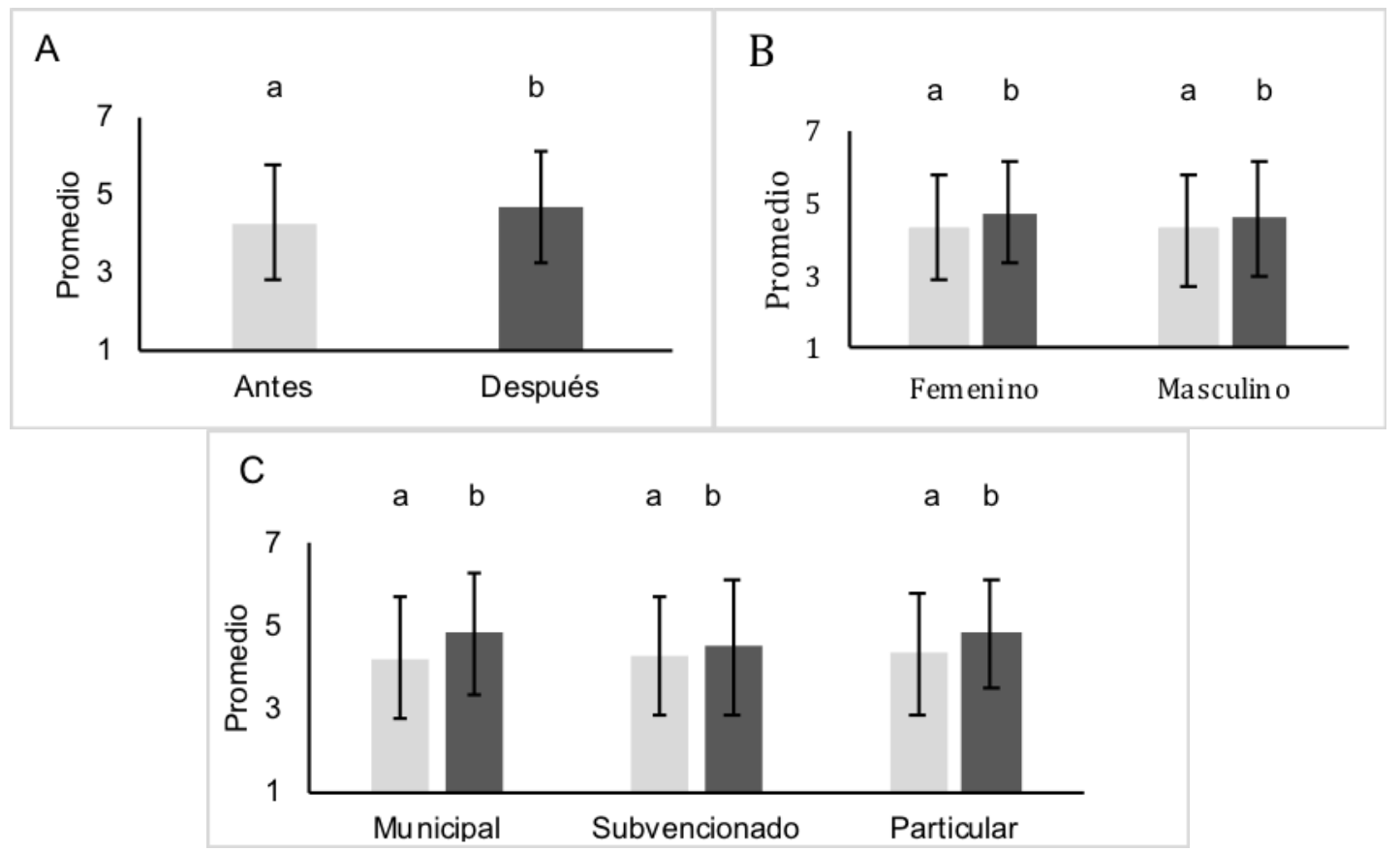

Fig. 2: Percepción de la complejidad de la ciencia declarada por los asistentes de la feria, antes (gris claro) y después (gris oscuro) del evento. Resultados (A) generales, (B) por género, y (C) tipo de establecimiento educacional. La escala mide cuán compleja es la ciencia para el asistente, donde $1=$ muy difícil y 7 = muy fácil. 


\section{Interés por la ciencia}

El interés general por la ciencia aumentó después de la feria $(t=4.38 ; P<0.0001 ; g l=1329)$ de $5.75+/-$ 1.66 a $6.11+/-1.33$. (Tabla 2, Figura 3). Esta tendencia se observó tanto en hombres como en mujeres ( $F=$ 23.35; $\mathrm{P}=<0.0001 ; \mathrm{gl}=1$ ) después de la feria. Los hombres subieron de $5.43+/-1.78$ a $5.96+/-1.36$, y las mujeres de $5.90+/-1.63$ a 6.20 +/- 1.31. Esta tendencia también se observó para los tres tipos de instituciones educativas que alcanzaron la feria $(F=21.83 ; P=<0.0001 ; \mathrm{gl}=1)$. Las escuelas públicas aumentaron de $5.49+/-1.95$ a $6.04+/-1.48$, mientras que las escuelas subsidiadas de $5.89+/-1.49$ a 6.14 +/- 1.21, y las escuelas privadas de 5.43 +/- 1.69 a 6.12 +/- 1.39. Estos resultados reiteran lo descrito por Retana y Vásquez (2016) respecto a los efectos de las ferias científicas de educación media en Costa Rica.

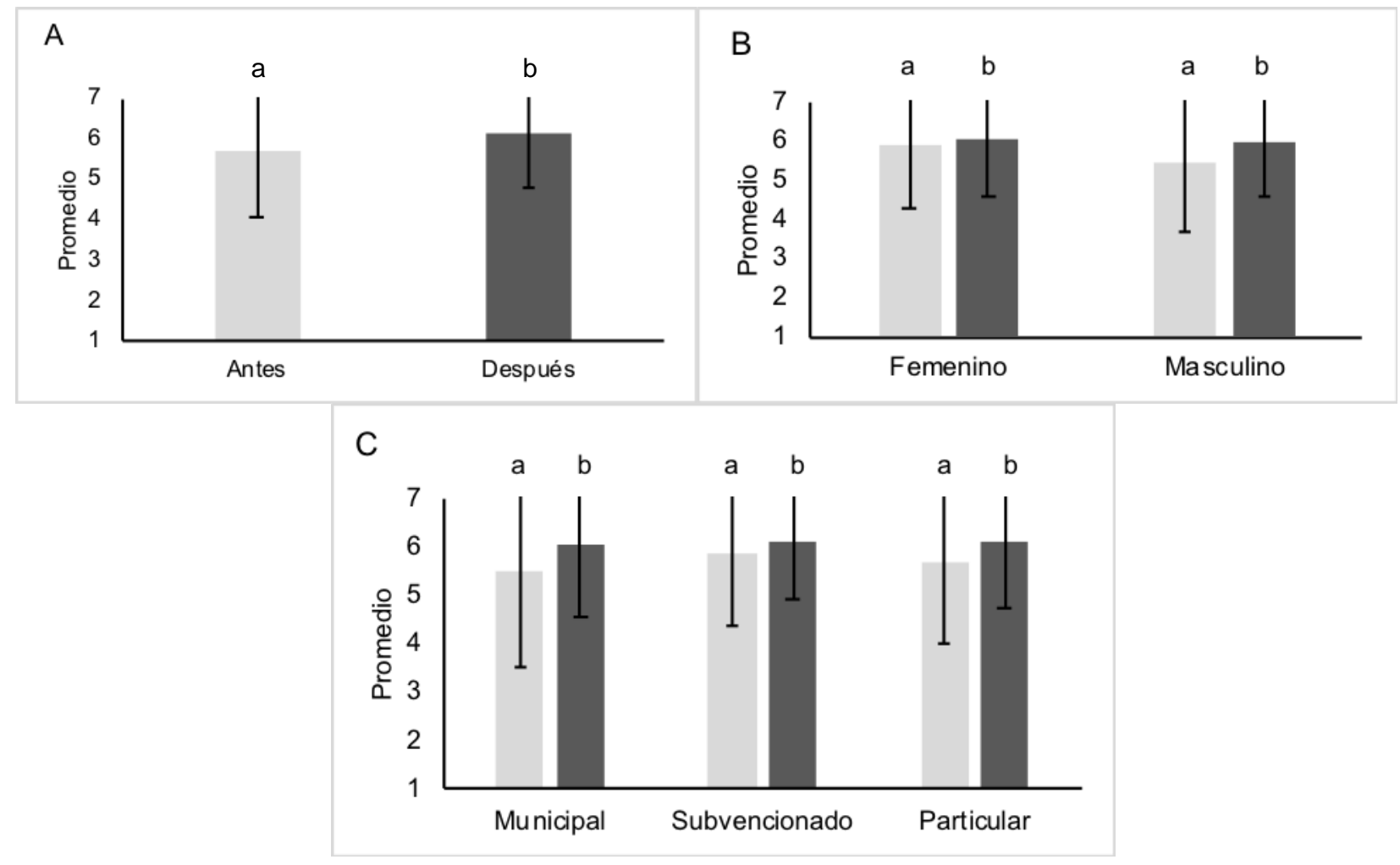

Fig. 3: Interés en seguir una carrera científica declarado por los asistentes de la feria, antes (gris claro) y después (gris oscuro) del evento. Resultados (A) generales, (B) por género, y (C) tipo de establecimiento educacional. La escala mide el interés por seguir una carrera de ciencia por el asistente, donde $1=$ nada de interés y $7=$ mucho interés.

Los resultados muestran que la motivación por la ciencia aumentó después de la feria, mientras que la percepción de su complejidad disminuyó. Estos dos hechos llevaron a un mayor interés por seguir una carrera científica después de la exposición a la feria. Los resultados reiteran la evidencia previa, en la que se plantea que las experiencias a corto plazo, como lo son eventos de educación informal como ferias y exposiciones, generan más motivación en el aprendizaje (Salmi, 1993; Retana et al., 2018).

Resulta interesante que no se obtuvieran diferencias de género para los resultados de este estudio, como ha sido tradicional y sistemáticamente reportado en estudios anteriores (Debacker y Nelson, 2000; Clark, 2005; Miller et al. 2006; Archer et al., 2013; Archer et al. 2014; Vásquez y Manassero, 2015), donde los hombres siempre muestran mayor motivación e interés por seguir carreras científicas que las mujeres de educación media.

Más interesante aún es el hecho de que no hubo diferencias en los resultados de los estudiantes de distintos establecimientos educacionales que asistieron a la feria, lo que se contrapone a muchos estudios previos (Goyette y Mullen, 2005; Padilla y Figueroa, 2015; Dabney et al., 2016). Este es un punto importante a remarcar, pues todas las iniciativas públicas de divulgación de la ciencia, a nivel nacional, privilegian instituciones de educación pública, muchas veces marginando y descuidando establecimientos educacionales subvencionados y privados. También es importante mencionar que muchas instituciones de educación pública, en Chile, orientan a sus estudiantes a seguir una educación técnico profesional, mostrando secundariamente las opciones científico-humanistas como carreras opcionales futuras (Lagos y Palacios, 2008). 


\section{Componentes de la feria}

Los estudiantes apreciaron de manera diferente los distintos tipos de stands exhibidos en la feria $(F=46.58$; $\mathrm{P}=<0.0001 ; \mathrm{gl}=4)$. Los más apreciados por los estudiantes fueron los de indagación científica, que obtuvieron $6.45+/$ - 0.96. En la segunda posición se clasificaron los afiches y los juegos con $6.13+/-1.18$ y $5.99+/-1.24$ respectivamente. En la tercera posición se ubicaron las sesiones de discusión con $5.89+/-$ 1.49. Finalmente quedaron los modelos 3D con $5.47+/-1.57$ (Tabla 1, Figura 4).

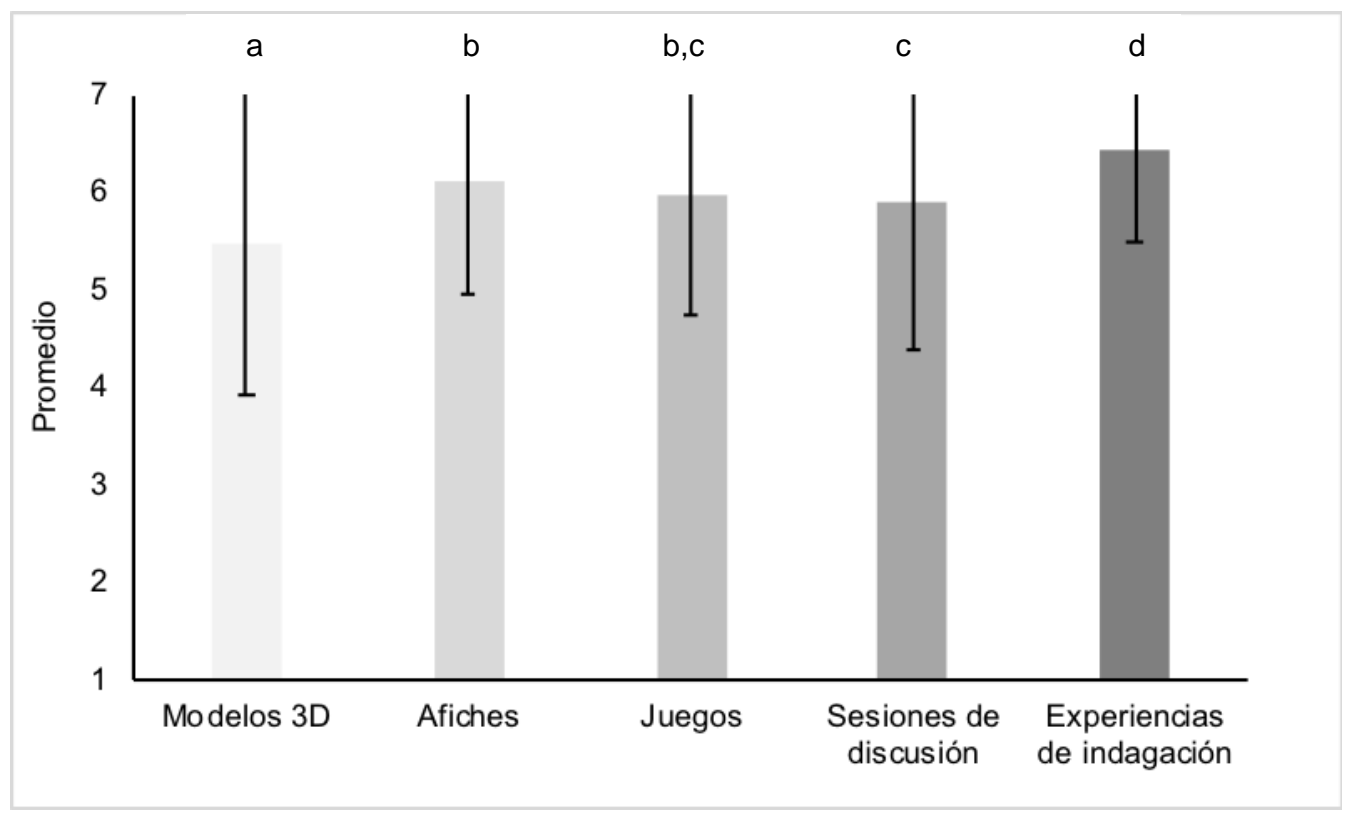

Fig. 4: Evaluación de los componentes de la feria de parte de los asistentes. Las letras (a,b,c y d) muestran diferencias entre los formatos.

Globalmente, los resultados muestran que los cinco formatos de stands de la feria recibieron buenas evaluaciones promedio de parte de los asistentes. La calificación promedio más baja, de 5.47 +/- 1.57 para modelos 3D, podría reflejar poca o la menor interacción entre monitores y asistentes de los formatos presentes en la feria. Por el contrario, la calificación más alta, de 6.45 +/- 0.96 para los stands de experiencias de indagación, podría reflejar una alta interacción entre monitores y asistentes, pues cada experiencia requería de constantes instrucciones y supervisión para la correcta implementación de experimentos y comprensión de resultados científicos obtenidos. Estos últimos resultados pueden ser considerados positivos, pues la indagación científica es de las metodologías más recomendadas en el proceso de aprendizaje de ciencias hace ya varias décadas (Gibson y Chase, 2002; Luehmann, 2009). Lo anterior podría indicar que se debe potenciar o priorizar el formato de indagación de ciencias en las ferias de ciencia a nivel escolar.

Relativo a la satisfacción general de los asistentes a la feria científica, el 99,7\% de los asistentes declaró que recomienda esta feria a sus pares. Este resultado, sumado a las altas notas promedio de todos los formatos de stands de la feria, nos permite plantear que hay una clara apreciación positiva de la feria por practicamente la totalidad de los asistentes al evento.

\section{Percepción de la ciencia y de los científicos}

Los resultados de la percepción de la feria para el concepto "ciencia" antes y después del evento comprenden un total de 2085 y 1888 palabras, respectivamente. Las diez palabras más frecuentes en la prueba a priori fueron: 1) Química (8.2\%), 2) Biología (8.1\%), 3) Experimento (7.8\%), 4) Física (7\%), 5) Cell $(4.4 \%), 6)$ Investigación (4.1\%), 7) Vida (3.4\%), 8) Átomo (3.2\%), 9) Naturaleza (2.2\%) y 10) Descubrimiento (1.7\%). Y las diez palabras más frecuentes en la prueba posterior fue: 1) Química (9\%), 2) Biología (8.8\%), 3) Física (7.9\%), 4) Experimento (6.9\%), 5) Investigación (4,4\%), 6) Célula y vida (3,3\%), 7) Átomo (2,4\%), 8) Descubrimiento (2,2\%) y 9) Naturaleza (2\%). Estos resultados se presentan en la Figura 5, en la que el tamaño de la palabra es proporcional a su frecuencia. 


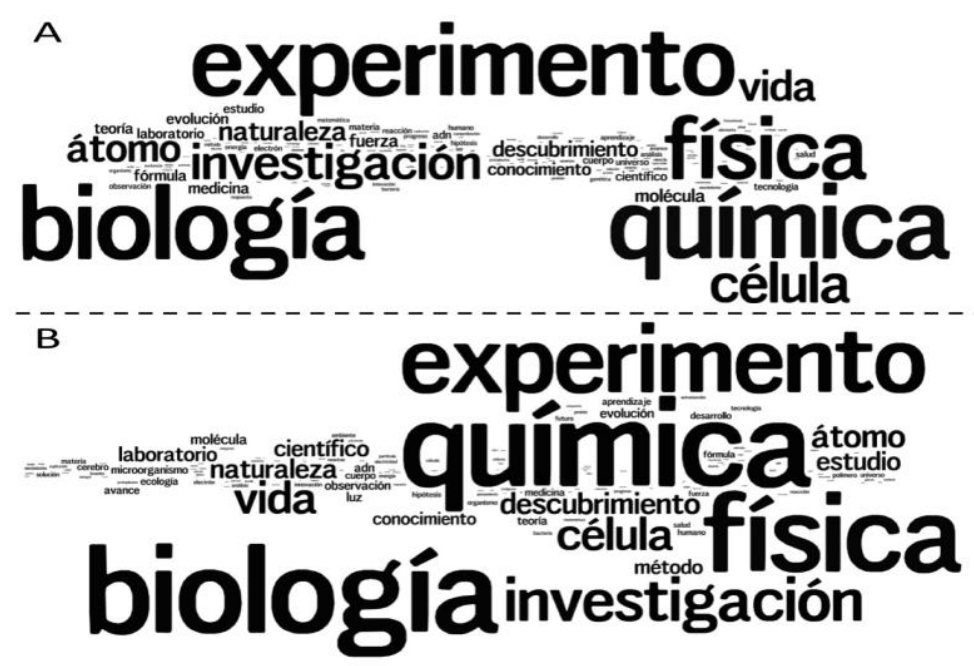

Fig. 5: Diagrama de la percepción de los asistentes relacionado con el término "ciencia" $(A)$ antes, y (B) después de la feria. El tamaño de las palabras es proporcional a su frecuencia.

Los resultados de percepción para el concepto "científico" antes y después de la feria comprenden un total de 1964 y 1819 palabras, respectivamente. Las diez palabras más frecuentes en la prueba a priori fue: 1) Experimentación (10.8\%), 2) Investigador (7.2\%), 3) Laboratorio (6.3\%), 4) Inteligente (5.1\%), 5) Estudioso $(2,7 \%), 6)$ Descubridor $(2,3 \%), 7)$ Método $(2,1 \%), 8)$ Médico $(1,8 \%)$ y 9$)$ Hipótesis y 10$)$ Biología $(1,6 \%)$. Y las diez palabras más frecuentes en la prueba posterior fue: 1) Experimentación (10.3\%), 2) Investigador e Inteligente (6.3\%), 3) Laboratorio (5.3\%), 4) Estudioso (3.1\%), 5) Curioso y biólogo (2,5\%), 6) Método $(2,3 \%), 7)$ Hipótesis $(2,2 \%), 8)$ Médico $(2 \%)$ y 9 ) Descubridor (1,3\%). Estos resultados se presentan en la Figura 6 , en la que el tamaño de la palabra es proporcional a su frecuencia.

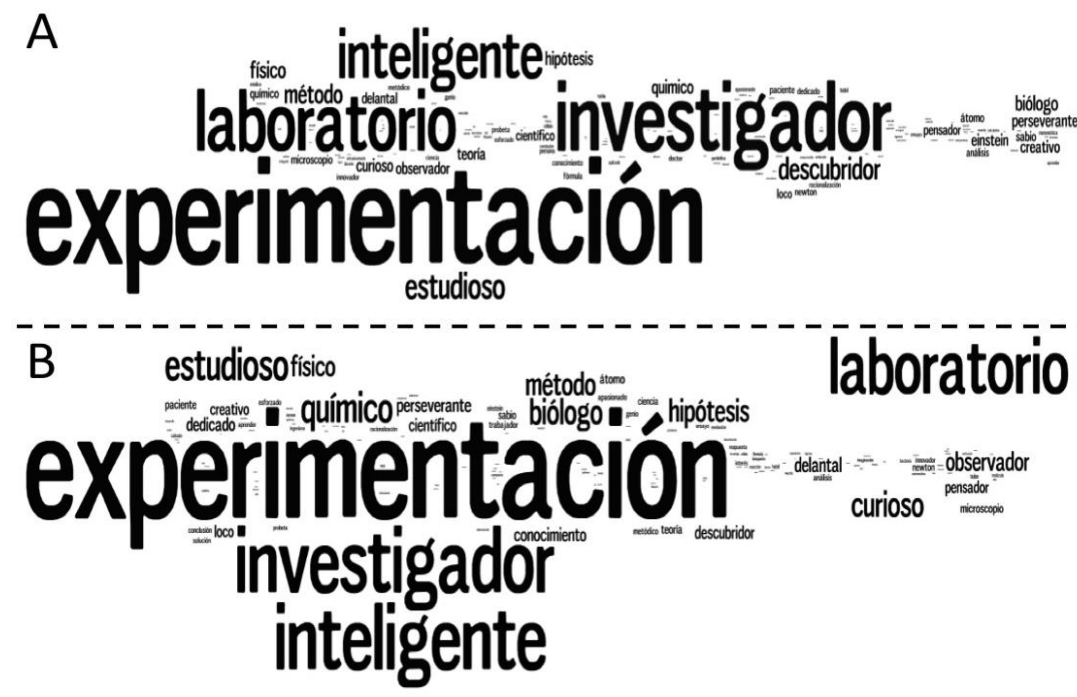

Fig. 6: Diagrama de la percepción de los asistentes relacionado con el término "científico" (A) antes, y (B) después de la feria. El tamaño de las palabras es proporcional a su frecuencia.

Ambos resultados, tanto los obtenidos para la percepción de "ciencia" como para la percepción de "científico", no muestran cambios trascendentales debido a la feria científica. Esto ratifica que una experiencia de corto plazo, como esta iniciativa, no permite por sí sola generar los cambios socioculturales de apreciación de las ciencias y los científicos. Será necesaria una sumatoria de actividades de corto plazo, o bien un programa de largo plazo, para generar cambios profundos en las percepciones de la cultura científica, y así contar con ciudadanos bien informados científicamente.

Es importante mencionar que el presente estudio presenta ciertas limitaciones en cuanto a su diseño. Relativo a los asistentes, cada establecimiento educacional utilizó un criterio diferente para traer a sus alumnos. Algunos colegios trajeron a cursos completos, mentras que otros colegios trajeron una selección de estudiantes ya sea como recompensa por buena conducta o bien aquellos que presentaron interés previo por la ciencia. También no fue posible discriminar si los asistentes visitaron la feria sólo para timbrar su pasaporte o bien para descubrir los distintos stands científicos, motivados por la ciencia. Respecto del 
número de encuestas respondidas, el $100 \%$ del test a priori no fue recibido, dado que estas debían venir firmadas por los apoderados y muchos asistentes lo olvidaron. Y para las encuestas a posteriori, un número menor respondió, dado que muchos asistentes pasaron demasiado tiempo en alguno(s) de los stands discutiendo con monitores sin tener tiempo de responder la encuesta de salida. Esto último permitió ver que el tiempo otorgado para visitar la feria resultó estrecho.

\section{CONCLUSIONES}

De acuerdo con los resultados de este estudio, y de su discusión y análisis, se pueden extraer las siguientes conclusiones principales: 1) las ferias científicas aumentan la motivación por la ciencia, el interés por seguir una carrera científica y disminuyen la percepción de la complejidad científica, en estudiantes de educación media, independientemente de su género y nivel socioeconómico. 2) El formato de stand más apreciado en esta ocasión fue el de experiencias de indagación científica, y el menos apreciado fue el de modelos 3D. Además de una clara apreciación positiva del formato general de la feria y sus componentes. 3) La percepción de los conceptos "ciencia" y "científico" varía ligeramente en frecuencia luego de esta experiencia a corto plazo.

\section{AGRADECIMIENTOS}

Agradecemos a la Vicerrectoría de Investigación y a las facultades de ciencia de la Pontificia Universidad Católica de Chile, así como a CONICYT PIA/BASAL FB002 por el financiamiento proporcionado para realizar la feria, al PAR Explora Norte y la Sociedad de Instrucción Primaria (SIP) por su convocatoria, a los monitores voluntarios que lideraron los stands de la feria, y a Daniella Mella por sus comentarios sobre el documento.

\section{REFERENCIAS}

Araújo, A., Feira de Ciências: Contribuiçoes para a Alfabetizaçao Científica na Educaçao Básica, Disertación doctoral, Universidad Federal do Ceará, Brasil (2015)

Archer, L., J. DeWitt y J. Dillon, 'It didn't really change my opinion': Exploring what Works, what Doesn't and why in a School Science, Technology, Engineering and Mathematics Careers Intervention, doi: 10.1080/02635143.2013.865601, Res. Sci.Technol. Educ., 32(1), 35-55 (2014)

Archer, L., J. DeWitt, y otros cuatro autores, Not Girly, not Sexy, not Glamorous': Primary School Girls' and Parents' Constructions of Science Aspirations, doi: 10.1080/14681366.2012.748676, Pedag. Cult. Soc., 21(1), 171-194 (2013)

Bencze, J.L. y G.M. Bowen, A National Science Fair: Exhibition Support for the Knowledge Economy, doi: 10.1080/09500690802398127, Int. J. Sci. Educ., 31(8), 2459-2483 (2009)

Chaparro, L., J.M. Acosta y otros catorce autores, Libro Verde Ferias de la Ciencia, (en la web: https://www.fecyt.es/es/publicacion/libro-verde-de-las-ferias-de-ciencia, acceso el 10 de abril de 2019), España (2018)

Clark, J., Women and Science Careers: Leaky Pipeline or Gender Filter?, doi: 10.1080/09540250500145072, Gender Edu., 17:4, 369-386 (2005)

Dabney, K.P., R.H. Tai y M.R. Scott, Informal Science: Family Education, Experiences, and Initial Interest in Science, doi: 10.1080/21548455.2015.1058990, Int. J. Sci. Educ., Part B, 6(3), 263-282 (2016)

Debacker, T.K. y R.M. Nelson, Motivation to Learn Science: Differences Related to Gender, Class Type, and Ability, doi: 10.1080/00220670009598713, J. Educ. Res., 93:4, 245-254 (2000)

Diamond, J., M.S. Horn y D.H. Uttal, Practical Evaluation Guide: Tools for Museums and Other Informal Educational Settings, 3rd Ed., Rowman \& Littlefield Publishers, Inc., Maryland, United States of America (2016)

Doran, G.T., There's a S.M.A.R.T. Way to Write Management's Goals and Objectives, Manag. Rev., 70 (11): $35-36$ (1981)

Falk, J. y L. Dierking, The 95 percent Solution: School is Not where Most Americans Learn Most of their Science, doi: 10.1511/2010.87.486, Am. Sci., 98 (6), 486-493 (2010)

Fariña, L.M., B. Gallardo y otros cuatro autores, Decálogo de la Comunicación de la Ciencia chilensis (en la web: http://heureka-online.com/opinion/condicion-necesaria/conocimiento-lujo-ilustrados, acceso 29 de noviembre de 2018), Chile (2018)

Finnerty, V., Can Participation in A School Science Fair Improve Middle School Students' Attitudes Toward Science and Interest in Science Careers, Unpublished doctoral dissertation, University of Massachusetts Lowell, United States of America (2013)

Gibson, H.L. y C. Chase, Longitudinal Impact of an Inquiry-based Science Program on Middle School Students' Attitudes toward Science, doi: 10.1002/sce.10039, Sci. Educ., 86, 693-705 (2002) 
Goyette, K.A. y A.L. Mullen, Who Studies the Arts and Sciences? Social Background and the Choice and Consequences of Undergraduate Field of Study, doi: 10.1080/00221546.2006.11778936, J. High. Educ., 77(3), 497-538 (2005)

Jeffs, T. y J. Ord, Rethinking Outdoor, Experiential and Informal Education: Beyond the Confines, $1^{\text {st }}$ Ed., 40-55, Routledge, London, England (2018)

Lagos, F. y F. Palacios, Orientación Vocacional y Profesional en Colegios de Bajo Nivel Socioeconómico, doi: 10.31619/caledu.n28.209, Cal. Edu., 204-244 (2008)

Luehmann, A. y D. Markowitz, Science Teachers' Perceived Benefits of an Out-of-school Enrichment Programme: Identity needs and university affordances, doi: 10.1080/09500690600944429. Int. J. Sci. Edu., 29(9), 1133-1161 (2007)

Luehmann, A.L., Students' Perspectives of a Science Enrichment Programme: Out-of-school Inquiry as Access, doi: 10.1080/09500690802354195, Int. J. Sci. Edu., 31(13), 1831-1855 (2009)

Markowitz, D.G., Evaluation of the Long-Term Impact of a University High School Summer Science Program on Students' Interest and Perceived Abilities in Science, doi: 10.1023/B:JOST.0000045467.67907.7b, J. Sci. Edu.Techno., 13(3), 395-407 (2004)

Miller, P.H., J. Slawinski y S. Schwartz, Gender Differences in High-school Students' Views about Science, doi: 10.1080/09500690500277664, Int. J. Sci. Edu., 28(4), 363-381 (2006)

Padilla, L.E. y A.E. Figueroa, Variables Socio-familiares Presentes en la Transición de los Egresados del Bachillerato a la Educación Superior en el Estado de Aguascalientes y su Elección de Carrera e Institución, doi: 10.33064/33crscsh, 560, Caleidoscopio, 33 (2015)

Ray, E., Outreach, Engagement Will Keep Academia Relevant to Twenty-First Century Societies, https://eric.ed.gov/?id=EJ589783, ISSN: ISSN-1086-2447, J. Pub. Serve. Out., 4(1), $21-27$ (1999)

Retana, D.A. y B. Vásquez, Ferias de Ciencia y Tecnología de Costa Rica: una Experiencia que Motiva la Elección de Carreras Científicas y Tecnológicas, ISSN: 02139529, Campo Abierto, 35(1), 13-30 (2016)

Retana, D.A., B. Vásquez y M.M. Camacho, Las Ferias de Ciencia y Tecnología en Costa Rica y sus Aportes a la Educación Secundaria, doi: https://doi.org/10.15517/aie.v18i2.33170, Actual. Invest. Edu., 18(2), 309-352 (2018)

Salmi, H., Science Centre Education. Motivation and Learning in Informal Education. Research Report; Doctoral Dissertation, University of Helsinki, Department of Teacher Education, Finland (1993)

Stern, M.J, R.B. Powell y D. Hill, Environmental Education Program Evaluation in the New Millennium: what do we Measure and what Have we Learned?, doi: 10.1080/13504622.2013.838749, Env. Edu. Res., 20(5), 581-611 (2014)

Vásquez, A. y M.A. Manassero, La Elección de Estudios Superiores Científico-técnicos: Análisis de algunos Factores Determinantes en Seis Países, doi: http://dx.doi.org/10.1590/1516-731320160020008, R.Eur.E.D.C. 12 (2), $264-277$ (2015) 
